\title{
Finite Element analysis of a shape memory alloy actuator for a micropump
}

\author{
Tarek Merzouki $^{\mathrm{a}, *}$, Arnaud Duval ${ }^{\mathrm{b}}$, Tarak Ben Zineb ${ }^{\mathrm{c}, \mathrm{d}}$ \\ a PRISME EA4229, Université d'Orléans, 45072 Orléans, France \\ ${ }^{\mathrm{b}}$ LaMCoS, INSA Lyon/CNRS, 69621 Villeurbanne, France \\ c Université de Lorraine, LEMTA, UMR 7563, Vandoeuvre-lés-Nancy F-54500, France \\ ${ }^{\mathrm{d}}$ CNRS, LEMTA, UMR 7563, Vandoeuvre-lés-Nancy F-54500, France
}

\begin{abstract}
This paper deals with a Finite Element (FE) behavior analysis of a shape memory alloy actu-ator for a micropump. It is composed of two membranes of NiTi shape memory alloy (SMA) in a martensitic state at room temperature. They have an initial flat shape and are bonded together with an intermediate spacer. The thermal loading allows the actuator to move up and down in the membrane normal direction. A detailed analysis of sensibility to material and geometric parameters of the SMA actuator is undertaken by FE method. The actuation capability and reliability are studied in order to lead to optimal parameters set providing a higher stroke (deflection) with a low heating temperature. The shape memory effect exhib-ited by these membranes is simulated by means of the phenomenological constitutive law based on Chemisky-Duval model [1,2], and implemented in the Abaqus ${ }^{\circledR}$ FE code. The obtained numerical results were detailed proving the ability of the proposed modeling to reproduce the actuator behavior under thermal loading. This analysis showed that it is possible to provide a large stroke for a minimal geometry of the actuator.
\end{abstract}

\section{Introduction}

Micromachining technology is a miniaturization technique which can be used to manufacture various devices and systems. This technique has opened a new thrusts and made the possibility to develop various devices and systems in small size with high functionality, precision and performance in various domains such as automotive, aerospace, communication, medical, civil engineering [3-7]. In fact, some microsystems require an effective actuator which could be suitable for highly sensitive applications. Smart materials as piezoelectric ceramics or shape memory alloys (SMAs) were often considered for the design of micro-components and/or micro-devices [8-17]. In fact, their coupled and multiphysic behaviors allow the conversion of an electric or thermal energy on a mechanical one for actuator applications and vice versa for sensor applications. The development of thin films elaboration processes for these smart materials combined by the using of etching techniques made possible the design of complex geometry micro-components and micro-devices (micro-actuators, micro-valves, micro-sensors, stents, etc.) based on theses thin films [18-25].

SMA actuators are ones of the most used micro-devices to achieve such applications. In fact, SMAs can exhibit high forces or large strains, which could be converted to high pressures or large strokes (deflection). For this reason, SMA actuators have been considered for micropump actuations [26-29] where the stroke of the actuator determines the pumped volume per cycle. The actuator converts the input electric or thermal energy into mechanical work output inducing system motion. A wide range of SMA actuators for micropumps and microvalves were proposed in the literature [14,21,30-37]. They were

\footnotetext{
* Corresponding author. Tel.: +33 (0)2 384943 58; fax: +33 (0)2 38417329 .

E-mail address: tarek.merzouki@univ-orleans.fr (T. Merzouki).
} 
etched in an SMA thin films (generally in NiTi) and they consider the shape memory effect property. During a heating, the actuator exhibits a displacement induced by the reverse martensitic transformation. This displacement is recovered during the cooling process leading to the forward martensitic transformation. This cooling process is generally very low (A fan can provide compressive convection for fast cooling [38]). For that reason, an actuator composed of two NiTi SMA membranes etched in a thin film and separated by a silicon spacer was proposed [30,31]. These two membranes are working in an antagonist way and the actuation is always obtained by heating. The heating of one membrane gives an actuation displacement in one way (fluid input) where the heating of the other membrane allows the opposite actuation displacement (fluid output). During the heating of any membrane, the other one is freely cooled. Thanks to the spacer inserted between membranes, the forward and reverse transformations are occurring under bending stress. This induces the activation and disappearance of oriented martensite variants leading to a significant transformation strain.

To analyze the behavior of such actuators, it is interesting to turn ones attention to many experimental investigations $[32,30,39-41]$ and numerical studies $[28,29,42,43]$ on various considered technologies in the case of micropumps. They are based on various experimental and/or numerical (FE simulation) tools in order to optimise their performance. In the present work, a numerical approach based on a finite element method (FEM) was considered. Most commercial codes based on FEM have a standard constitutive models database well adapted to conventional materials with elastic, viscoelastic, elasticplastic, elasticviscoplastic, etc. behaviors. However, these codes have very few or no constitutive models that describes all the specificities of an SMA thermomechanical behavior. As an example the FEM based Abaqus code has a superelastic constitutive law based on the Auricchio's model $[44,45]$. In order to analyse by FEM the shape memory effect behavior of such actuators, the only solution was to consider a user constitutive model which can be implemented in commercial FEM based code via a user subroutine (as an example one can use the User MATerial (UMAT) subroutine for Abaqus). The LEMTA's and the LEM3's (affiliated to Université de Lorraine, Arts et Mtiers ParisTech and CNRS) research groups have, in collaboration, developed and implemented in Abaqus a thermomechanical constitutive law covering all the specificities of the SMA behavior (superelasticity, shape memory effect, internal loop behavior, difference between tension compression, saturation and hysteresis effects, etc.) $[1,46]$. In this model the inelastic strain induced by forward and reverse transformations is characterised by four internal variables. Two are scalar and correspond, respectively, to the volume fraction of all the martensite and the twinned part. The two other internal variables are tensorial and correspond respectively to the mean transformation and the mean twinning strains. The constitutive equations of this model were derived from an assumed expressions of the Gibbs free-energy and the dissipation function. The driving forces corresponding to internal and control variables were derived and compared to the corresponding yield forces in order to determine active mechanisms. Additional developments allowed to take into account the asymmetry between tension and compression, the management of internal loop and the saturation behaviors. This FE analysis considered the 2D version of this constitutive model corresponding to the plane stress assumption.

An analysis of the influence of the various material and geometric parameters was discussed. The aim is to highlight factors in relation with the actuator stroke. Finally, an optimal configuration was suggested able to provide a high stroke with minimal geometry. The optimization objective function was the actuation capability and reliability of the actuator. In some cases, the optimization and a higher heating temperature can lead to a higher actuator stroke. On the other hand, this leads to a higher stress level and an important transformation strain in the NiTi actuator. In that case, the actuator becomes unreliable to operate if the stress and transformation strain is beyond material strength. In order to ensure both high stroke and reliability, a corresponding criterion was defined. The obtained results allowed designing the configuration of the proposed actuator with an optimized stroke.

This paper is organized into five sections including the introduction and the conclusion. In the second section, the description of the SMA actuator properties was detailed. The studied material is a NiTi SMA in the martensitic state with a selfaccommodate martensite variants at room temperature. The third section deals with the adopted constitutive model and the numerical simulation of the SMA actuator behavior based on a FEM. Finally, the sensitivity to material and geometrical parameters on the stroke of the actuator was discussed. The FE analysis gave a significant results of the actuator behavior under a thermal loading. Results allowed defining a set of geometrical parameters for the actuator providing an important stroke with a minimal size.

\section{Description of the studied SMA actuator}

The actuator adopted design was initially proposed by Benard et al. [30,31]. It considers two NiTi membranes at the martensitic state with a self-accommodate martensite variants at room temperature as shown in Fig. 1a. They have an initial flat shape and are bonded together with a crown in the exterior contour (exterior spacer), and with an intermediate spacer in silicon material. This assembly induces a bending stress field on membranes leading to an orientated martensite and a strain state recoverable by thermal loading. When the top membrane is heated, it becomes austenitic and recovers the deformation, thus the actuator moves downwards, resulting a further deflection in the other membrane. The cooling allows it to return to a martensitic state. When the bottom membrane is heated, it becomes austenitic and causes the deformation, so the actuator moves in the other direction. The principle of the actuator is shown in Fig. 1. There are several possible actuation methods for such SMA actuator: 


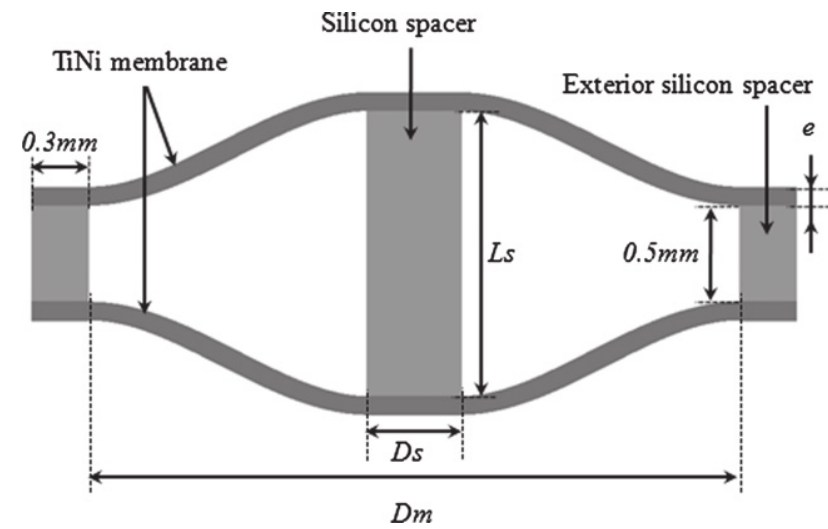

(a) Actuator, initial state

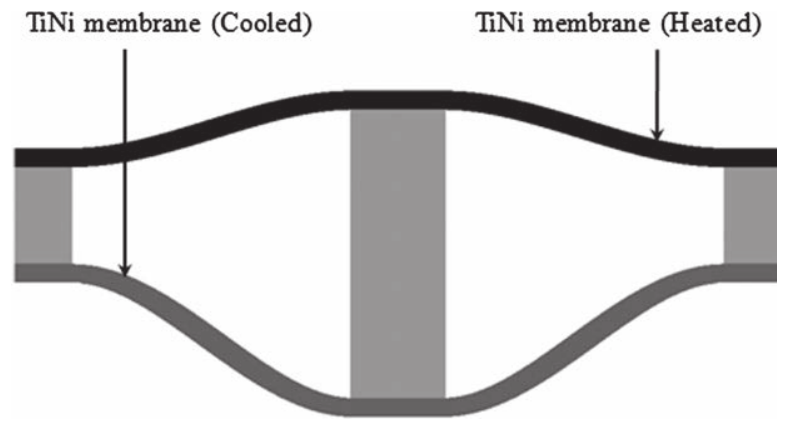

(b) After heating of the top membrane

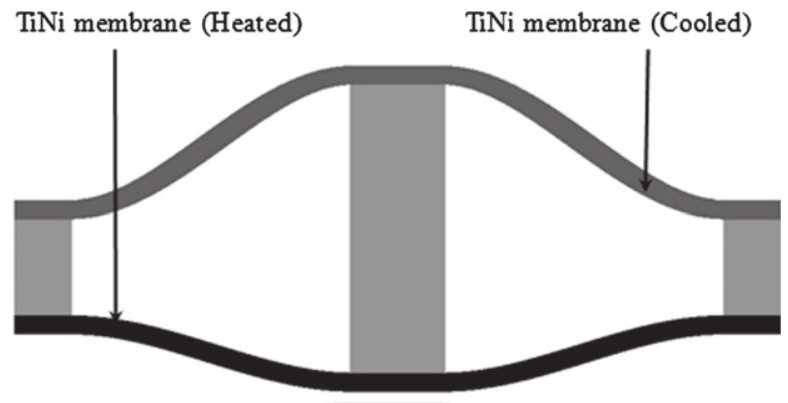

(c) After the heating of the bottom membrane

Fig. 1. Sketch of the NiTi actuator cross-section. Heating or cooling membrane causes the motion along the horizontal direction, $e$ is the membrane thickness, $D m$ is the membrane diameter, $D s$ is the spacer diameter, $L s$ is the spacer length.

(1) Heating and cooling of the SMA membranes [47]: there are two types to heat up SMA. One is by electrical current and depends heavily on electrical resistivity. The other is by heat transfer (by hot air/water or exposing to thermal radiation) and it relates to thermal conductivity which is ignored in the fast heating case [47]. It is mentioned in this reference that, the required temperature increasing for actuation depends on the set-up of SMA in terms of pre-strain and stress, hardening-like in transformation, environmental temperature, the required actuation temperature, etc.

(2) Depending on how bias pressure is applied: (a) pressurization, or (b) evacuation [32].

In the present work, the heating of SMA membranes was obtained by applying an electrical current, and the electrical resistivity of the SMA thin film is suitable for Joule heating. The cooling occurs under free convection condition in air. It is well known that the SMA actuator is able to provide high stroke with low frequency due to the slow response time during the cooling under free convection condition in air. For high frequency, additional cooling method is required, which is difficult to consider for micropump. A synthesis work on the heating/cooling of the SMA was presented in [8,47,48]. In conclusion, for the considered actuator with two SMA membranes, the two way displacement is always obtained by heating which is easier to control than cooling.

\section{Finite Element analysis of SMA actuator behavior}

In this section, the adopted SMA constitutive law and the FE model of the above mentioned SMA actuator are detailed.

\subsection{SMA thermomechanical behavior model}

The adopted behavior model is based on a thermodynamical description of the martensitic phase transformation, reorientation and detwinning. Four internal variables were considered. Two are scalar and define the martensitic volume fraction in the considered representative volume element and the volume fraction of the twinned martensite. The two others internal 
variables describe, in an averaged way, the orientation of martensite and the accommodation of twins, respectively. Assuming small perturbations (framework of infinitesimal strains), the additive decomposition of the total strain is then written as:

$$
\varepsilon=\varepsilon^{e}+\varepsilon^{t h}+\varepsilon^{T}+\varepsilon^{t w i n}
$$

where $\boldsymbol{\varepsilon}^{\boldsymbol{e}}$ is the elastic strain, $\boldsymbol{\varepsilon}^{\text {th }}$ is the thermal expansion strain, $\boldsymbol{\varepsilon}^{\boldsymbol{T}}$ is the inelastic strain due to martensitic transformation, $\varepsilon^{t w i n}$ is the inelastic strain due to the accommodation of twins between martensite variants.

Transformation strain can be expressed as the average value over the RVE of local transformation strain field:

$$
\boldsymbol{\varepsilon}^{T}=\frac{1}{V} \int_{V} \boldsymbol{\varepsilon}^{T}(r) d V=\frac{V_{M}}{V} \frac{1}{V_{M}} \int_{V_{M}} \boldsymbol{\varepsilon}^{T}(r) d V=f \overline{\boldsymbol{\varepsilon}}^{T}
$$

where $f$ denotes the martensitic volume fraction, and $\overline{\boldsymbol{\varepsilon}}^{\mathrm{T}}$ denotes the mean transformation strain over the martensite volume.

The strain resulting from the inelastic accommodation of twins between martensite variants is written:

$$
\boldsymbol{\varepsilon}^{t w i n}=\frac{1}{V} \int_{V} \boldsymbol{\varepsilon}^{t w i n}(r) d V=\frac{V_{M}^{F A}}{V} \frac{1}{V_{M}^{F A}} \int_{V_{M}} \boldsymbol{\varepsilon}^{t w i n}(r) d V=f^{F A} \overline{\boldsymbol{\varepsilon}}^{t w i n}
$$

where $f^{F A}$ denotes the formed self-accommodated martensitic volume fraction and $\overline{\boldsymbol{\varepsilon}}^{\text {twin }}$ denotes the mean twins accommodation strain over the martensitic volume. According to the expression of different strain mechanisms, one obtains:

$$
\boldsymbol{\varepsilon}=\boldsymbol{S}: \boldsymbol{\sigma}+\boldsymbol{\alpha}\left(T-T_{r e f}\right) \delta+f \overline{\boldsymbol{\varepsilon}}^{\boldsymbol{T}}+f^{F A} \overline{\boldsymbol{\varepsilon}}^{\text {twin }}
$$

where $\boldsymbol{S}$ is the isotropic fourth order compliance tensor, $\boldsymbol{\sigma}$ is the mean stress over the representative volume element (RVE), $\boldsymbol{\alpha}$ is the isotropic thermal expansion tensor, and $T_{r e f}$ is the reference temperature where thermal expansion strain is selected to be null.

A Gibbs' free energy potential was defined, including contributions of chemical, mechanical and interaction between martensite variants and interactions between grains energies.

$$
\begin{aligned}
G & =U^{A}-T S^{A}+B\left(T-T_{0}\right) f-\frac{1}{2} \boldsymbol{\sigma}: \boldsymbol{S}: \boldsymbol{\sigma}-\boldsymbol{\sigma}: \boldsymbol{\alpha} \Delta T-\boldsymbol{\sigma}: \overline{\boldsymbol{\varepsilon}}^{T} f-\boldsymbol{\sigma}: \overline{\boldsymbol{\varepsilon}}^{\mathrm{twin}} f^{F A}+\frac{1}{2} H_{f} f^{2}+\frac{1}{2} f H_{\varepsilon} \overline{\boldsymbol{\varepsilon}}^{T}: \overline{\boldsymbol{\varepsilon}}^{\boldsymbol{T}}+\frac{1}{2} f^{F A} H_{t w i n} \overline{\boldsymbol{\varepsilon}}^{\text {twin }} \\
& : \overline{\boldsymbol{\varepsilon}}^{\mathrm{twin}}+C_{v}\left[\left(T-T_{0}\right)-T \log \frac{T}{T_{0}}\right]
\end{aligned}
$$

where $U^{A}$ is the internal energy of the austenitic phase, $T$ is the current temperature, $S^{A}$ is the entropy of the austenitic phase. $B$ is the difference between entropies of austenite and martensite, it is also related to the stress-temperature slope of transformation limits. $T_{0}$ is the thermodynamical equilibrium temperature between austenite and martensite phases. $H_{f}$ is the pseudo-hardening coefficient of phase transformation, $H_{\varepsilon}$ and $H_{t w i n}$ are two material parameters describing interactions coming from phase transformation evolution and geometrical incompatibilities. $C_{v}$ is the latent heat.

The Clausius-Duhem inequality was written in terms of the Gibbs free energy:

$$
-\frac{\partial G}{\partial \boldsymbol{\sigma}}: \dot{\boldsymbol{\sigma}}-\frac{\partial G}{\partial T}: \dot{T}-\frac{\partial G}{\partial \boldsymbol{V}_{\boldsymbol{k}}}: \dot{\boldsymbol{V}}_{\boldsymbol{k}}-\dot{\boldsymbol{\sigma}}: \boldsymbol{\varepsilon}-\vec{q} \cdot \frac{\overrightarrow{\operatorname{gradT}}}{T} \geqslant 0
$$

The Gibbs free energy defined by Eq. (5) was considered as a thermodynamical potential defined as a function of state variables, internal variables and material constants depending on the considered media. The driving forces corresponding to these internal variables are derived from this expression.

$$
\begin{aligned}
& A_{f}=\frac{\partial G}{\partial f}=B\left(T-T_{0}\right) f-\boldsymbol{\sigma}: \overline{\boldsymbol{\varepsilon}}^{T}-\xi^{F A} \boldsymbol{\sigma}: \overline{\boldsymbol{\varepsilon}}^{t w i n}+\frac{1}{2} H_{\varepsilon} \overline{\boldsymbol{\varepsilon}}^{T}: \overline{\boldsymbol{\varepsilon}}^{T}+\frac{1}{2} H_{f} f+\frac{1}{2} H_{t w i n} \overline{\boldsymbol{\varepsilon}}^{\text {twin }}: \overline{\boldsymbol{\varepsilon}}^{\text {twin }} \\
& A_{\overline{\boldsymbol{\varepsilon}}^{T}}=\frac{\partial G}{\partial \overline{\boldsymbol{\varepsilon}}^{\mathbf{T}}}=-f \boldsymbol{\sigma}^{\prime}+f H_{\varepsilon} \overline{\boldsymbol{\varepsilon}}^{T} \\
& A_{\overline{\boldsymbol{\varepsilon}}^{\text {twin }}}=\frac{\partial G}{\partial \overline{\boldsymbol{\varepsilon}}^{\text {twin }}}=-f^{F A} \boldsymbol{\sigma}^{\prime}+f^{F A} H_{t w i n} \overline{\boldsymbol{\varepsilon}}^{t \text { twin }}
\end{aligned}
$$

$\boldsymbol{\sigma}^{\prime}$ is the deviatoric part of the stress tensor $\boldsymbol{\sigma}$. Based on this thermodynamical forces, one can define a transformation criterion, an orientation criterion, twins accommodation criterion and build the state diagram. Then evolution laws of the internal variables are deduced from the consistency conditions of the activated mechanisms (transformation, orientation and accommodation of twins). The evolution law of $f^{f A}$ was defined by the following relation:

$$
\dot{f}^{F A}=\xi^{F A} \dot{f} \begin{cases}\frac{\varepsilon_{S A T}^{T}-\bar{\varepsilon}_{e q}^{T}}{\varepsilon_{S A T}^{T}} \dot{f}, & \dot{f}>0 \\ \frac{f_{r}^{F F}}{f_{r}} \dot{f}, & \dot{f}<0\end{cases}
$$

where $\varepsilon_{S A T}^{T}$ is a material parameter characterizing the maximum transformation strain. $\overline{\boldsymbol{\varepsilon}}_{e q}^{T}$ is the von Mises equivalent mean transformation strain. $f_{r}$ is the martensite volume fraction at the reversal. $f_{r}^{F A}$ is a self accommodated martensite present at the transformation reversal. 
Table 1

Adopted material parameters for simulation of the NiTi actuator [1].

\begin{tabular}{llllll}
\hline$E(\mathrm{MPa})$ & $v$ & $\alpha$ & $\varepsilon_{\text {tensile } \max }^{T}$ & $\varepsilon_{\text {comp max }}^{T}$ & $A_{f}\left({ }^{\circ} \mathrm{C}\right)$ \\
\hline 70000 & 0.3 & $1 . \mathrm{E}-05$ & 0.07 & 0.07 & 40 \\
$r_{f}$ & $F_{\varepsilon}(\mathrm{MPa})$ & $H_{f}(\mathrm{MPa})$ & $H_{\varepsilon^{T}}(\mathrm{MPa})$ & $H_{\text {twin }}(\mathrm{MPa})$ & 60 \\
\hline 0.3 & 150 & 4 & 1000 & 60000 \\
\hline
\end{tabular}

The non-symmetric behavior between tension and compression was introduced through a maximum mean transformation strain depending on the second and third stress invariants. The transformation strain direction is determined by a normality flow-rule assumption. All these considerations lead to nonlinear constitutive equations, solved by a NewtonRaphson algorithm. More details about specificities of this model (saturation and internal loop management, difference between tension and compression behaviors, etc.) can be found in [1,2]. This model, implemented into the Abaqus FE code via the User MATerial (UMAT) subroutine, is able to describe superelastic behavior, recovery stress and strain (useful to design actuators), martensite reorientation and shape memory effect. For a given loading increment, the UMAT updates stresses and internal variables values and compute the values of tangent operators. These are useful for Abaqus in order to compute the global equilibrium of the structure. This model presents a 3D and 2D-plane-stress versions. The last one was considered for this study.

Some adopted material parameters governing the SMA behavior were identified for a NiTi [1]. Whereas other parameters, were taken from literature (their values did not vanish for standard NiTi). These parameters are: the Young's modulus $E$, poisson's ratio $v$, the thermal expansion $\alpha$, the maximum transformation strain in tension, $\varepsilon_{\text {tensile max }}^{T}$ and compression, $\varepsilon_{\text {comp max }}^{T}$, the martensite start temperature $M_{s}$, the austenite finish temperature $A_{f}$, the amplitude ratio of inner loops $r_{f}$, the stress reorientation of variants $F_{\varepsilon}$, the pseudo-hardening coefficient of phase transformation $H_{f}$, the two material parameters describing interactions coming from phase transformation evolution and geometrical incompatibilities $H_{\varepsilon T}$ and $H_{t w i n}$, and the Clausius-Clapeyron slope in the stress-temperature diagram $B$. Values of these material parameters are given in Table 1.

\subsection{Geometry, mesh and boundary conditions}

The geometric parameters are shown in Fig. 1a. The influence of this set of parameters on the actuator behavior is analyzed in Section 4. The initial shape of NiTi membranes is characterised by a $10 \mu \mathrm{m}$ thickness and a $3 \mathrm{~mm}$ diameter. The exterior wall is $0.3 \mathrm{~mm}$ of thickness. The intermediate spacer between the two membranes is $1.5 \mathrm{~mm}$ of length and of $0.5 \mathrm{~mm}$ diameter in silicon. All dimensional details of the actuator are given in Fig. 1. Once the geometry is introduced, the next part focuses on the sample meshing.

The study of the mesh refining is an important numerical aspect for the FE analysis. Indeed, an optimisation of the FE size meshing leads to reach a reliable description of stress-strain gradients. The NiTi membranes were meshed with coupled thermal-displacement shell elements with three and four nodes and linear interpolation (S4T, S3T). An elastic behavior with a high stiffness was assumed for the intermediate spacer and the exterior crown because they present only a rigid-body motion. They were meshed with hexahedral (C3D8) and tetrahedral (C3D6) solid continuum isoparametric elements with linear interpolation. These elements have selective integration method corresponding to a complete integration of the deviatoric tensor and a reduced integration of the hydrostatic one. An example of actuator meshing is shown in Fig. 2. The number of finite elements of each part of the micropump is given in the Table 2. Once the FE model is introduced, the SMA constitutive model is called by the FE code.

The applied loading path on the two membranes is shown in Fig. 3. The first step $(A B)$ corresponds to the martensite variants orientation process in the two NiTi membranes as presented in Fig. 3a. These membranes were bonded together with an intermediate spacer by applying a mechanical loading at room temperature lower than $M_{f}$. The second step (BC) corresponds to the thermal loading of membranes above the austenite finish temperature $A_{f}$. When the first membrane is freely cooled, the second one is heated as presented in Fig. 3b. Steps 0-1 and 1-2 correspond to the heating and cooling of the top membrane (continuous), the bottom one (dashed) is cooled at room temperature in this step. Steps 2-3 and 4-5 correspond to the heating and cooling of the bottom membrane where the top one is cooled. In this study, the room temperature and the heating level were fixed to $30^{\circ} \mathrm{C}$ and $100{ }^{\circ} \mathrm{C}$ respectively.

\subsection{Preliminary results and discussion}

Fig. 4a shows the actuator stroke during the steps $0-1,1-2$ and $2-3$ which were presented in Fig. $3 \mathrm{~b}$. Fig. $4 \mathrm{~b}$ gives the stroke as a function of applied temperature during the heating of the bottom membrane (step 2-3). The stroke increases with heating temperature increasing. It seems that the actuator starts to move at temperature about $50{ }^{\circ} \mathrm{C}$. It can be observed that when heating temperature reaches $70^{\circ} \mathrm{C}$, the stroke saturates to an asymptotic value, by continuing slightly increasing. This corresponds to full reverse transformation (martensite to austenite). When the NiTi material is subjected to higher stress and strain, plasticity occurs after an elastic domain of martensite and a failure is induced. Consequently, for reliability analysis of 


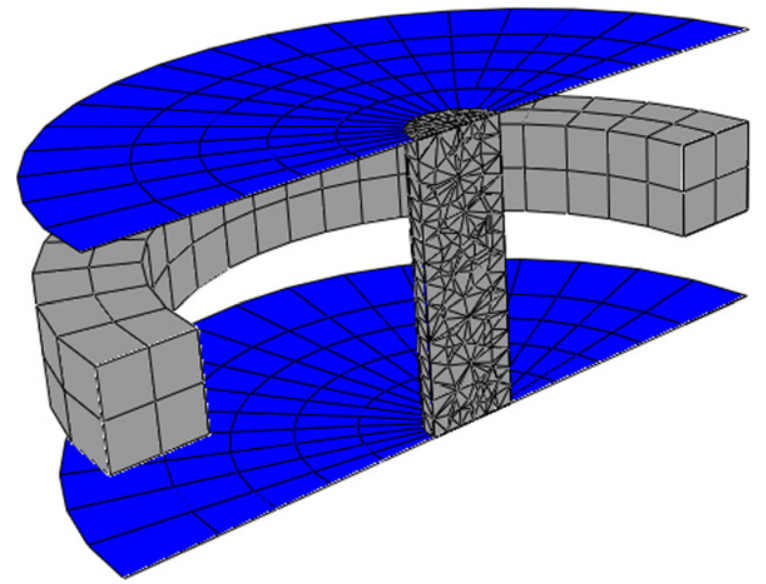

(a) FE model before the conditioning of the NiTi membranes

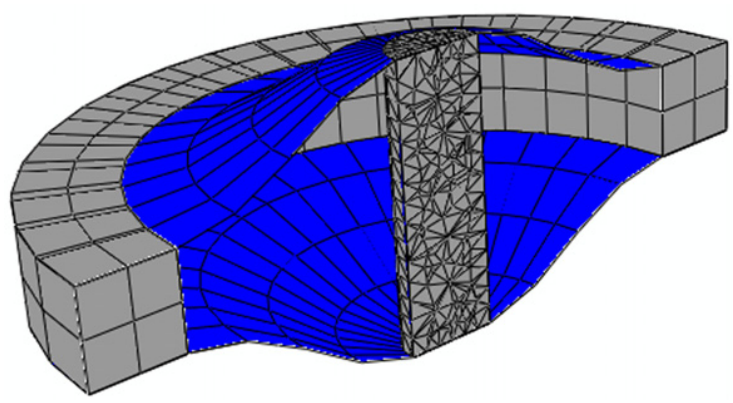

(b) FE model after the conditioning of the NiTi membranes

Fig. 2. FE model for the NiTi actuator.

Table 2

Number of finite elements of each part of the micropump.

\begin{tabular}{llll}
\hline & Intermediate spacer & Exterior crown & NiTi membranes \\
\hline Number of finite element & $1608(433$ nodes $)$ & $152(342$ nodes $)$ & $272 \times 2(291 \times 2$ nodes $)$ \\
\hline
\end{tabular}

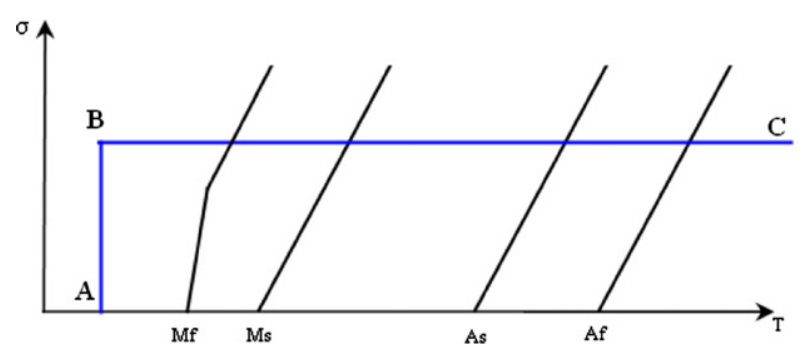

(a) Type of loading

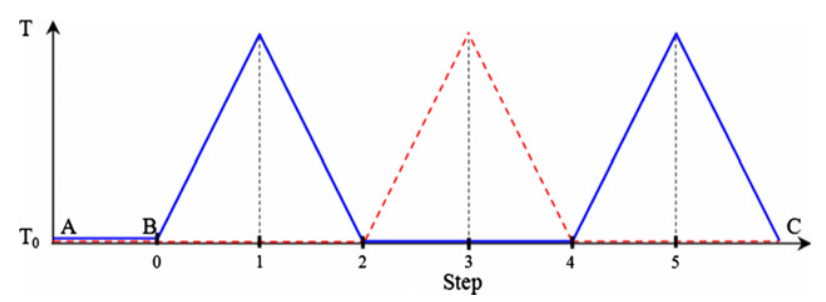

(b) Thermal loading, the continuous line corresponds to the top membrane, the dashed one corresponds to the bottom membrane

Fig. 3. Thermal loading for the two NiTi membranes.

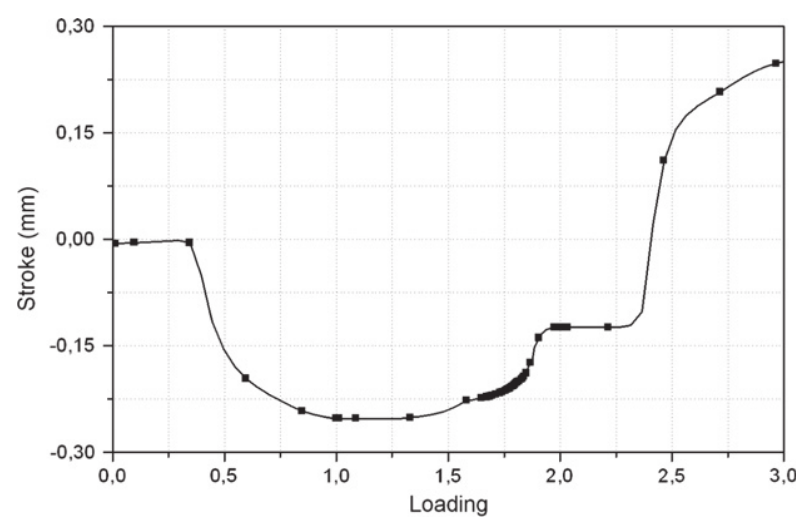

(a) Stroke during the steps $0-1,1-2,2-3$

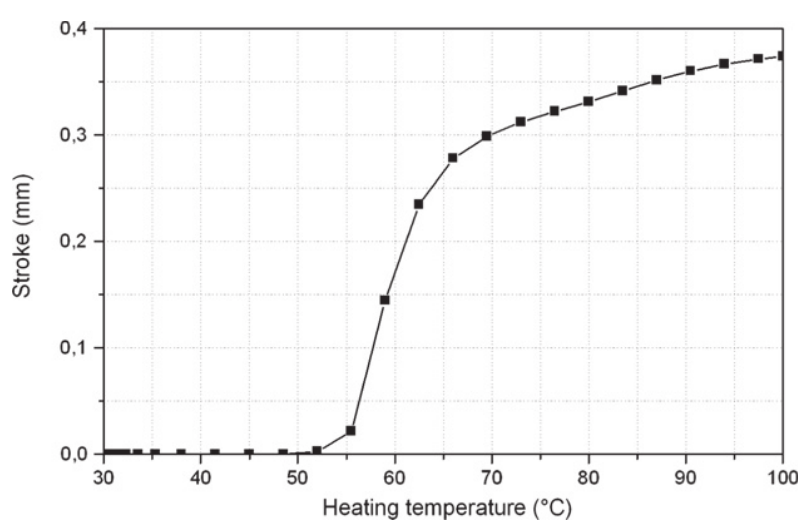

(b) Stroke as a function of heating temperature

Fig. 4. Stroke (deflection) of the actuator. 


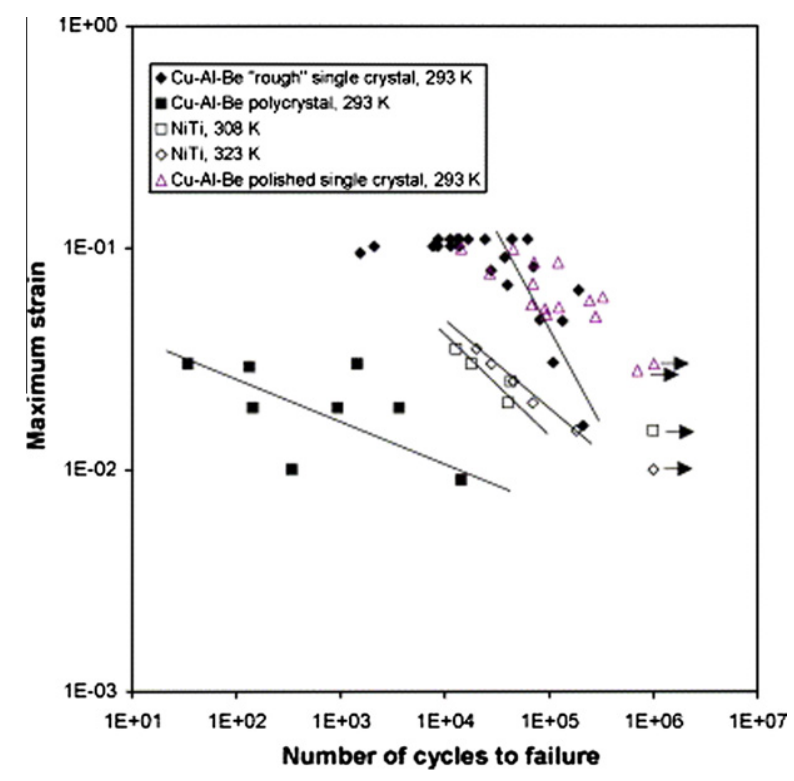

Fig. 5. Strain amplitude-fatigue life curves at various test temperatures [50].

NiTi, the maximum stress and transformation strain in NiTi should be below 500 MPa and 6.7\% [49] respectively. These constraints were adopted as a criterion during a reliability study. Following this condition, it can be concluded that the adopted maximum applied heating temperature is about $70^{\circ} \mathrm{C}$ (Fig. 4).

The NiTi SMA is used under cyclic loading after stabilizing its behavior by applying initial bending cycles. However, the thermal cyclic loading leads to fatigue behavior and, consequently, causes the loss of shape memory effect properties. Fig. 5 presents the number of cycles before failure versus the maximum imposed strain [50]. Results were compared on one hand with the ones obtained for polycrystal $\mathrm{Cu}-\mathrm{Al}-\mathrm{Be}$ wires and polished single crystal $\mathrm{Cu}-\mathrm{Al}-\mathrm{Be}$ wires, on the other hand, the NiTi polycrystal SMA in rotary bending tests are also compared. The strain decreases when the number of cycle increases. This figure highlights the importance to take into account the influence of the cyclic loading on the strain, and consequently on the actuator stroke. Therefore, it is necessary to remain at a low strain level so that the degradation of stroke remains weak. Fig. 5 allows also to investigate performances and reliability of the NiTi actuator during thermal cycling.

Fig. 6 presents the transformation equivalent strain at the step 3: the conditioning of the two NiTi membranes, the heating and the cooling of the top membrane. They correspond to steps $A-B, 0-1,1-2$, respectively. When the bottom membrane was heated or cooled, the obtained transformation equivalent strain state is similar to those in Fig. $6 \mathrm{~b}$ and b, respectively.

It is well known that the SMA actuator is able to provide high stroke with low frequency due to the slow response time during the air-cooling of the NiTi membrane. Hence, in the following section, a detailed analysis on the influence of the various material and geometric parameters was carried out. The objective was to find the sensitivity to parameters in order to lead to an optimized structural design corresponding to an actuator large deflection. It then provides a higher stroke with remaining in the reliability domain of the NiTi alloy. The stroke can be easily controlled by an applied heating loading path.

\section{Parameters influence}

This section studies the influence of the various possible parameters on the actuator deflection. For that, various simulations were carried out in the aim to predict the stroke variation, and to highlight its sensitivity to geometrical and material parameters. The FE model was set-up in Abaqus FE code, the geometry is presented in the Fig. 1. The material parameters are given in Table 1. For geometrical parameters, thermal loading and the martensite orientation start yield stress, three different values were considered in order to carry out their influence on the actuator behavior. For each step, the stroke variation, maximum von Mises stress and von Mises equivalent transformation strain in the NiTi actuator are presented. Finally, an optimal model in the studied parameters domain was proposed in term of high stroke with minimal geometry.

\subsection{Sensitivity to material and thermal loading parameters}

In this analysis, the geometry of the micropump is that given in Fig. 1 where values of dimensions are montioned in the Section 3. Studied parameters are the heating, and the martensite orientation start yield stress. 


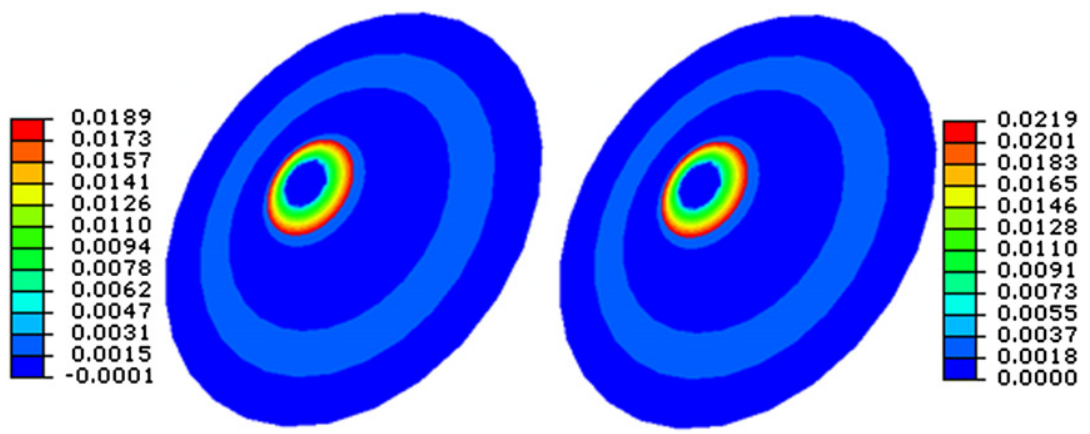

(a) After the conditioning of the two NiTi membranes

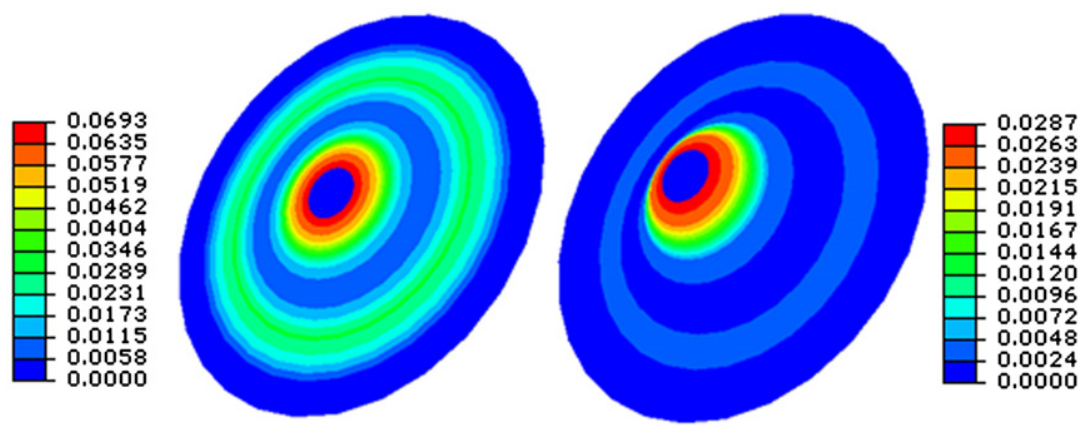

(b) After the heating of the top membrane

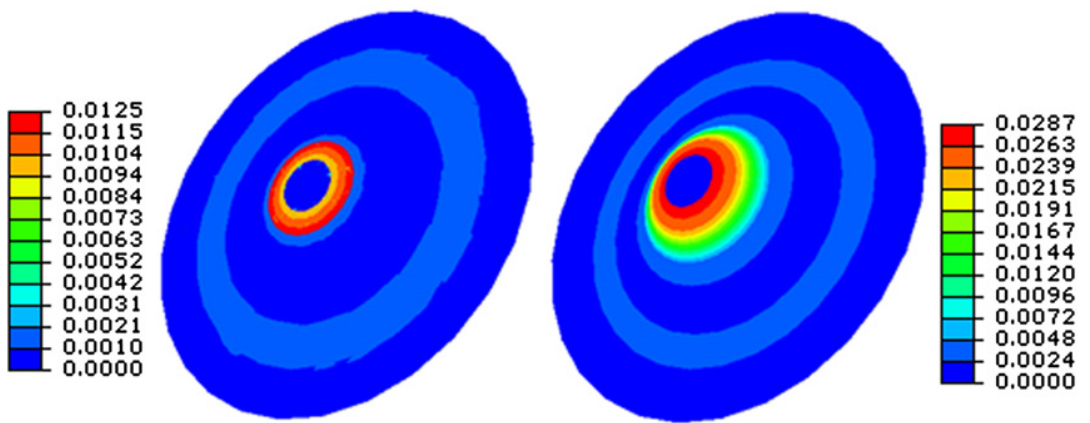

(c) After the cooling of the top membrane

Fig. 6. Transformation equivalent strain at the steps $A-B, 0-1,1-2$ (Fig. 3b), top membranes at the left and the bottom ones at the right.

\subsubsection{Heating level}

Fig. 7 presents the actuator stroke during the loading for three heating levels. It shows that the heating temperature plays an important role on the stroke. The stroke decreases with a heating level decreasing, and vice versa. It can be seen that there is a nonlinear relationship between the stroke and the heating level.

\subsubsection{Martensite orientation yield stress}

Figs. 8 and 9 present the actuator stroke, maximum von Mises stress and equivalent von Mises transformation strain during the heating for three values of the martensite orientation yield stress. These figures show that the stroke increases when this yield stress decreases. When this latter decreases, the self-accommodated martensite increases inducing a high deflection. There is a nonlinear relationship between the stroke and this yield stress corresponding to orientation activation. In order to remain in the reliability domain of NiTi material, the heating level must be stopped at $70{ }^{\circ} \mathrm{C}$. This heating level allows to decrease the response time during cooling of the NiTi membrane. An orientation start yield stress value of $100 \mathrm{MPa}$ corresponds to a maximal stroke. 


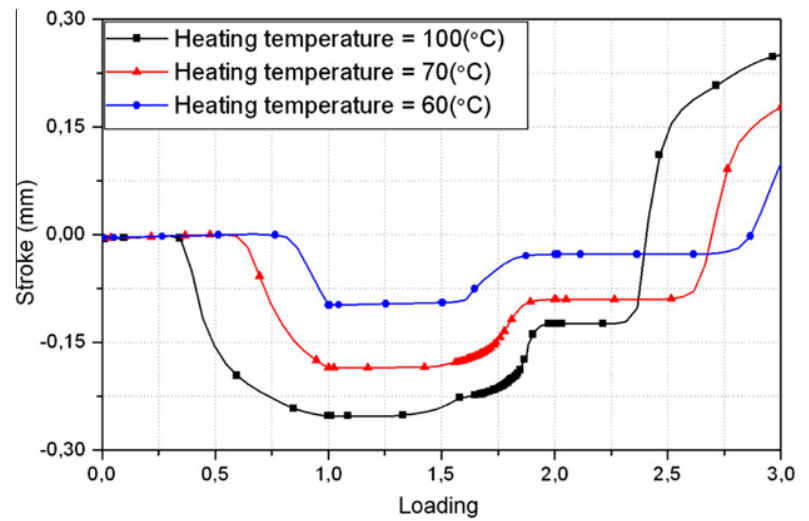

(a) Stroke

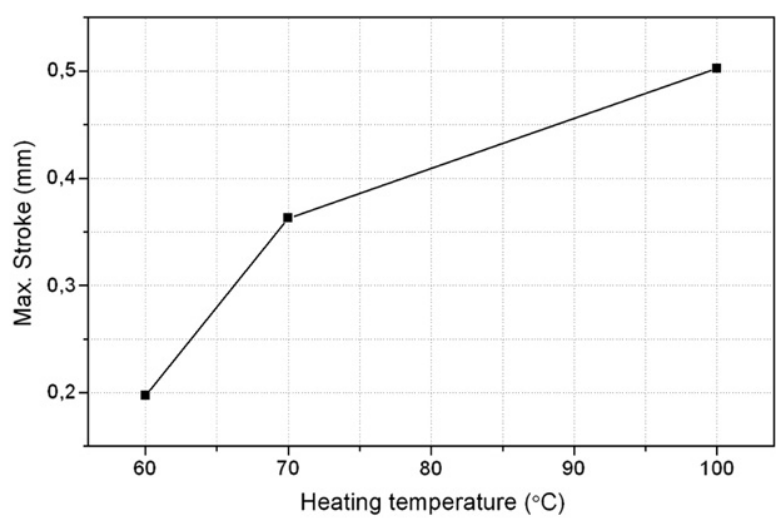

(b) Maximum stroke

Fig. 7. Stroke of the actuator during the steps $0-1,1-2,2-3$ and as a function of heating temperature (see Fig. $3 \mathrm{~b}$ ).

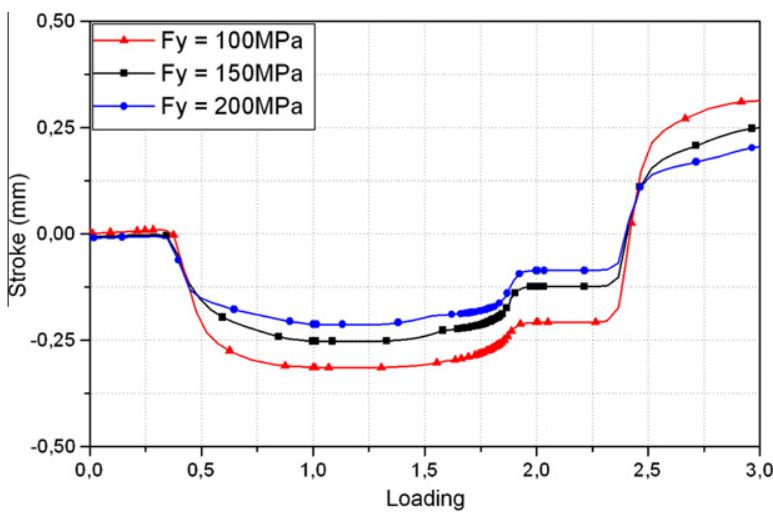

(a) Stroke

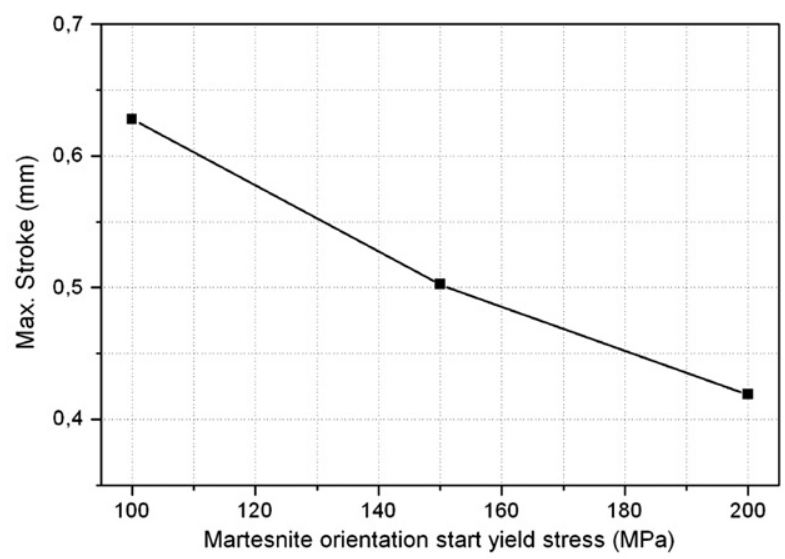

(b) Maximum stroke

Fig. 8. Stroke of the actuator during the steps $0-1,1-2$ and 2-3.

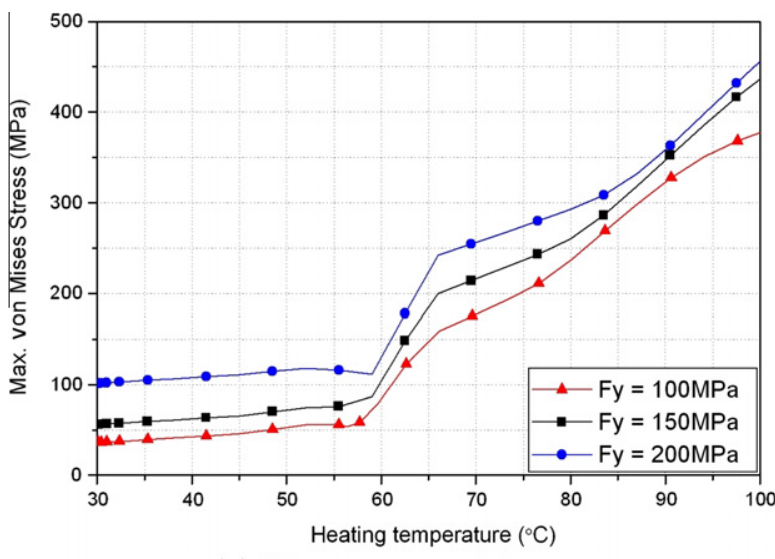

(a) Maximum von Mises stress

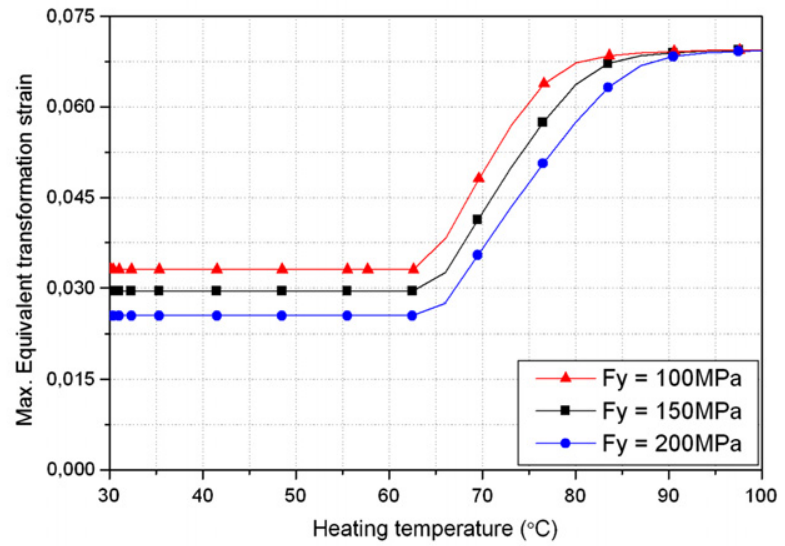

(b) Maximum equivalent transformation strain

Fig. 9. Maximum von Mises stress and equivalent transformation strain in the NiTi actuator as a function of heating temperature. 


\subsection{Sensitivity to geometrical parameters}

The following section is devoted to the analysis of geometric parameters effect on the actuator stroke. The material parameters were fixed and given in Table 1.

\subsubsection{Sensitivity to NiTi membrane thickness}

Figs. 10 and 11 present the same quantities for Figs. 8 and 9 for three values of the membranes thickness. The stroke increases when the thickness increases. It should be remarked that, when the membrane thickness increases, stiffness increases inducing a higher stress, the maximum transformation strain becomes significant, and consequently the deflection of the actuator is increased. For important thickness, large martensite self accommodation appears, with less martensite orientation, leading to a small variation of the transformation strain. There is a nonlinear relationship between the stroke and the thickness. For thicknesses of $30 \mu \mathrm{m}$ and $20 \mu \mathrm{m}$, the reliability criteria is not ensured. Moreover, for $30 \mu \mathrm{m}$ and $20 \mu \mathrm{m}$, the heating or cooling time is higher. However, in order to respect the reliability criterion, the heating level is fixed to $70^{\circ} \mathrm{C}$, and a membrane of $10 \mu \mathrm{m}$ thickness is adopted. The choice is also justified by an easier experimental procedure of assembly during the fabrication of the actuator.

\subsubsection{Sensitivity to NiTi membrane diameter}

Figs. 12 and 13 present the previous quantities for three values of the membranes diameter. The thickness of membranes was fixed to $10 \mu \mathrm{m}$. From the Fig. 12, the stroke decreases while the diameter increases. The increase of the membrane diameter produces a less global rigidity with inducing a reduction of stress, the maximum transformation strain was decreased,

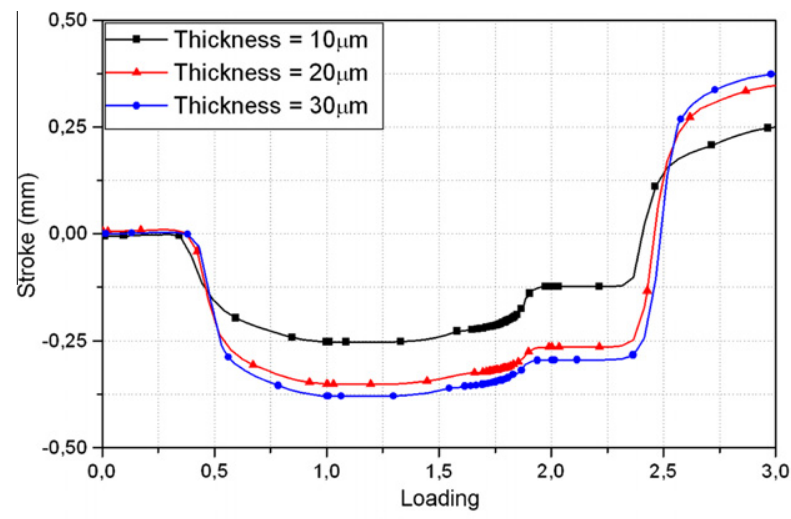

(a) Stroke

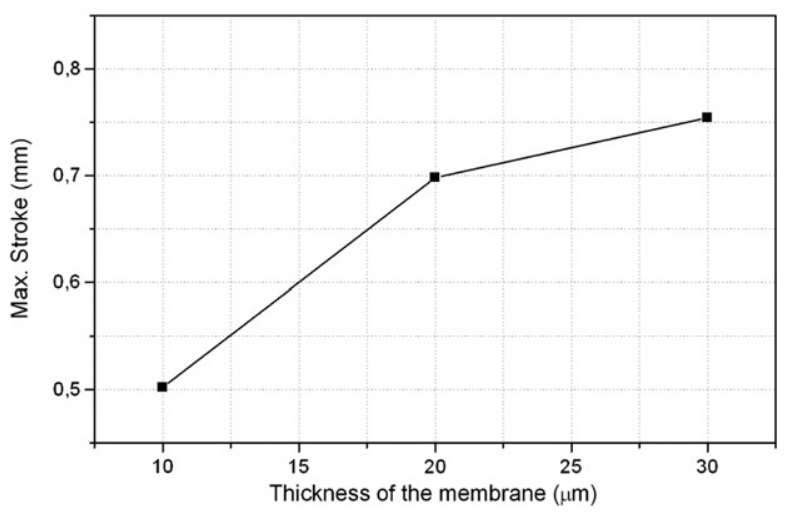

(b) Maximum stroke

Fig. 10. Stroke of the actuator during the steps $0-1,1-2$ and $2-3$.

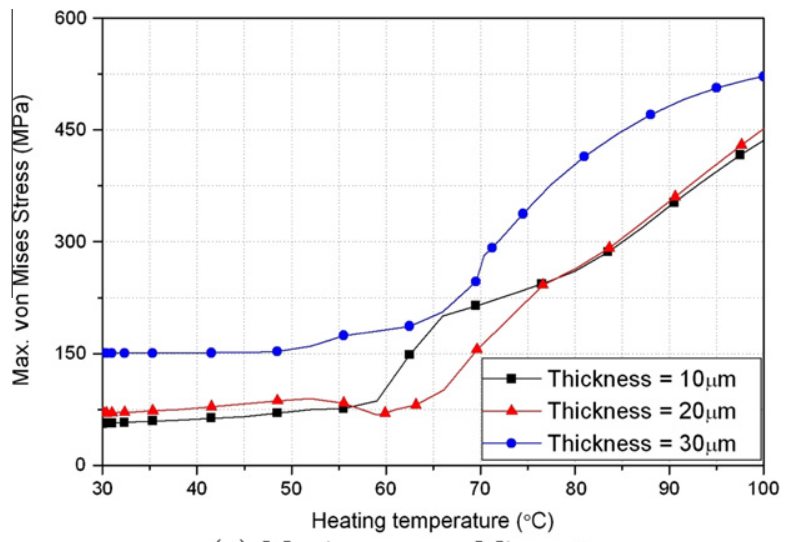

(a) Maximum von Mises stress

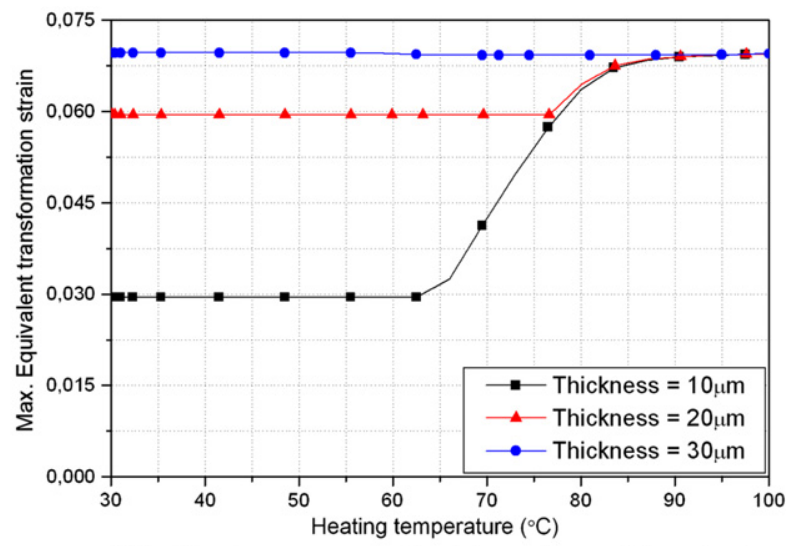

(b) Maximum equivalent transformation strain

Fig. 11. Maximum von Mises stress and equivalent transformation strain in the NiTi actuator as a function of heating temperature. 


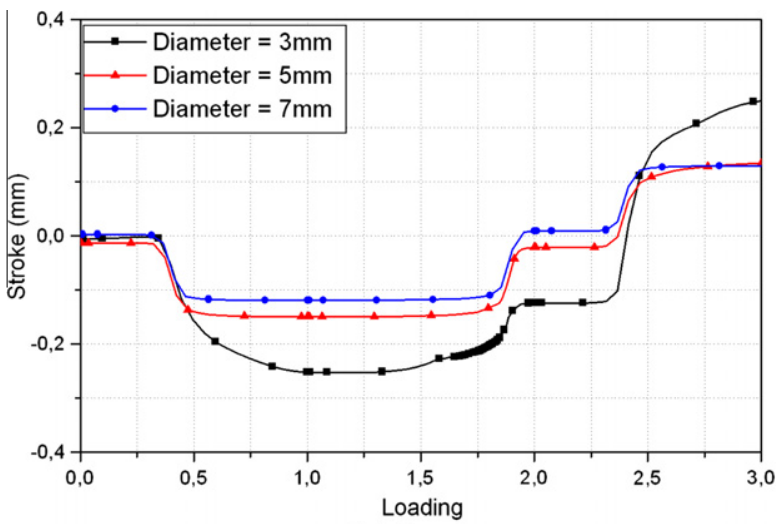

(a) Stroke

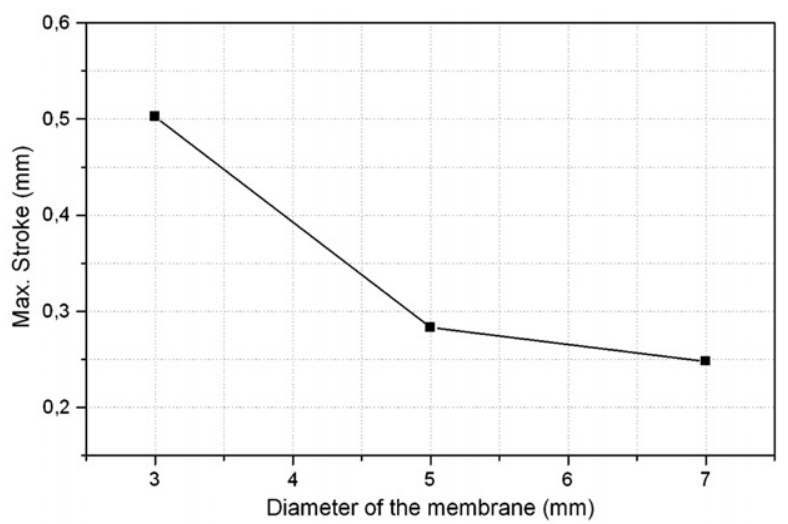

(b) Maximum stroke

Fig. 12. Stroke of the actuator during the steps $0-1,1-2$ and $2-3$.

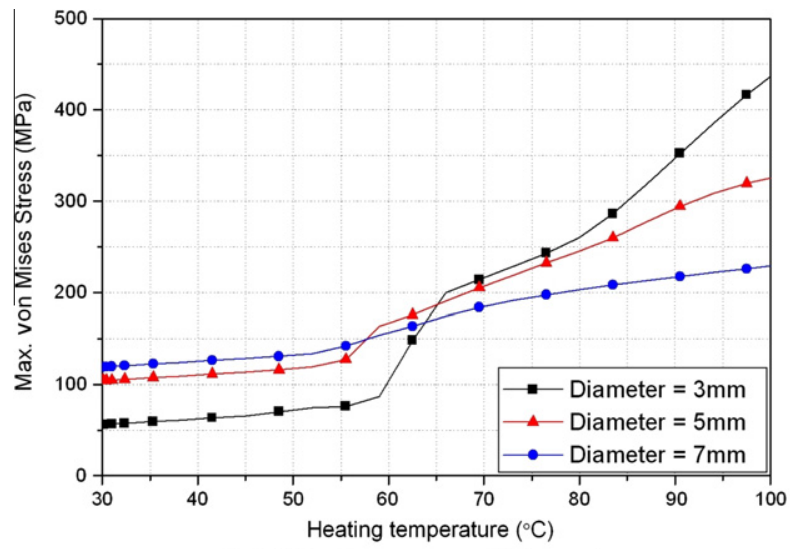

(a) Maximum von Mises stress

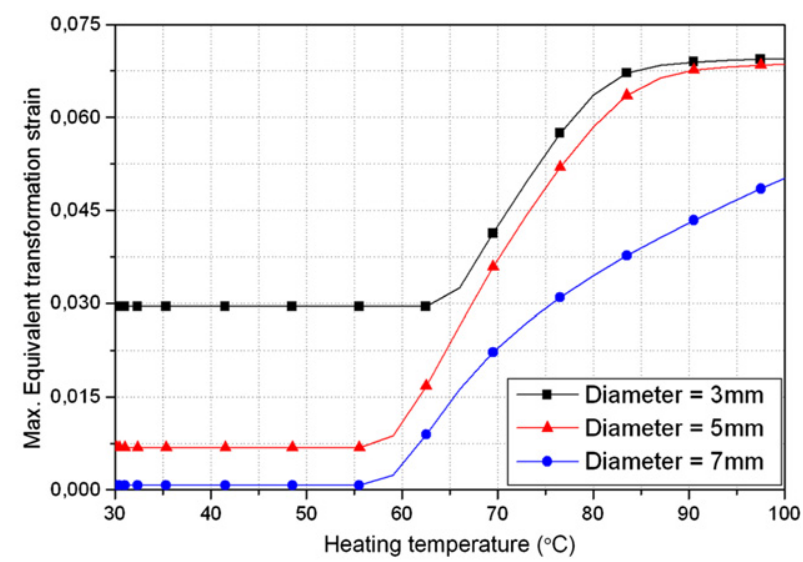

(b) Maximum equivalent transformation strain

Fig. 13. Maximum von Mises stress and equivalent transformation strain in the NiTi actuator as a function of heating temperature.

consequently the deflection of the actuator was reduced. For all these results, the reliability criterion was not ensured. In order to have an actuator of minimal geometry, two membranes with $3 \mathrm{~mm}$ of diameter were considered. The reliability criterion was respected when the heating level is stopped at $70^{\circ} \mathrm{C}$.

\subsubsection{Sensitivity to spacer diameter between the two membranes}

Figs. 14 and 15 present the previous quantities for three values of the spacer diameter. The thickness and diameter of membranes was fixed to $10 \mu \mathrm{m}$ and $3 \mathrm{~mm}$ respectively. The stroke increases when the diameter increases. There is a linear relationship between the stroke and the diameter. The experimental assembly of the two NiTi membranes may be difficult for the maximum value of diameter. But, due to difficulties in fabrication of such actuator with a spacer between two NiTi membranes, a diameter of $0.5 \mathrm{~mm}$ corresponds to a good compromise. So that, to check the reliability criterion, it is necessary to stop the heating level also at $70^{\circ} \mathrm{C}$.

\subsubsection{Sensitivity to spacer length between the two membranes}

Figs. 16 and 17 give the previous quantities for three values of the spacer length. The thickness and diameter of membranes were fixed respectively to $10 \mu \mathrm{m}$ and $3 \mathrm{~mm}$. The diameter of spacer was fixed to $0.5 \mathrm{~mm}$. The stroke increases with the spacer length increasing. The increase of the spacer length induces a high stress leading to a high martensite self-accommodation and low martensite orientation in the membrane. So the maximum transformation strain becomes important. There is a linear relationship between the stroke and the spacer length. In order to make possible the experimental assembly of the two membranes, to have an actuator with a small size geometry and to respect the reliability criterion, the heating level is stopped at $70^{\circ} \mathrm{C}$. The adopted compromise value of the length is $1.5 \mathrm{~mm}$. 


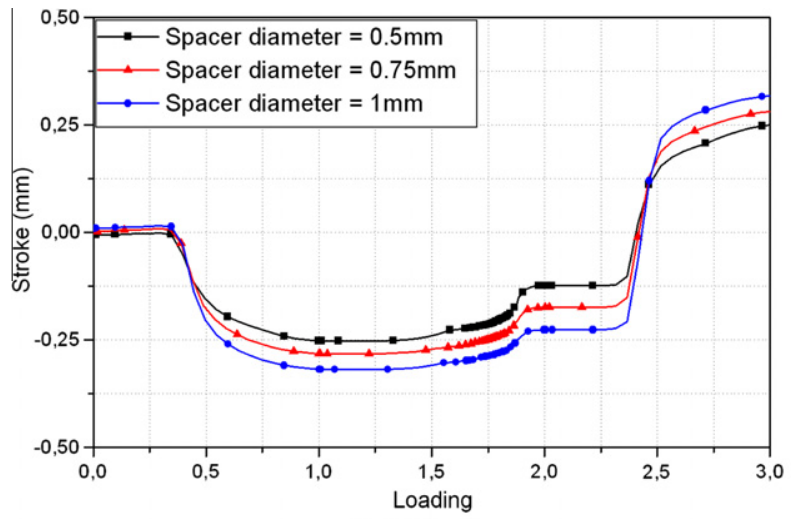

(a) Stroke

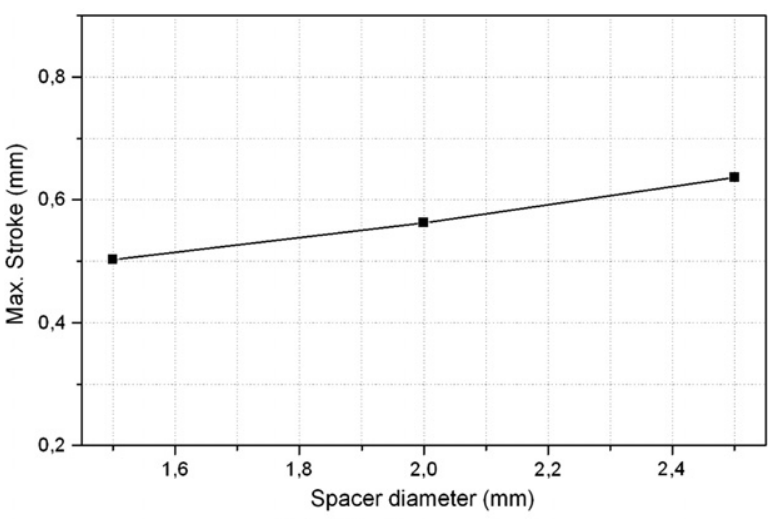

(b) Maximum stroke

Fig. 14. Stroke of the actuator during the steps $0-1,1-2$ and $2-3$.

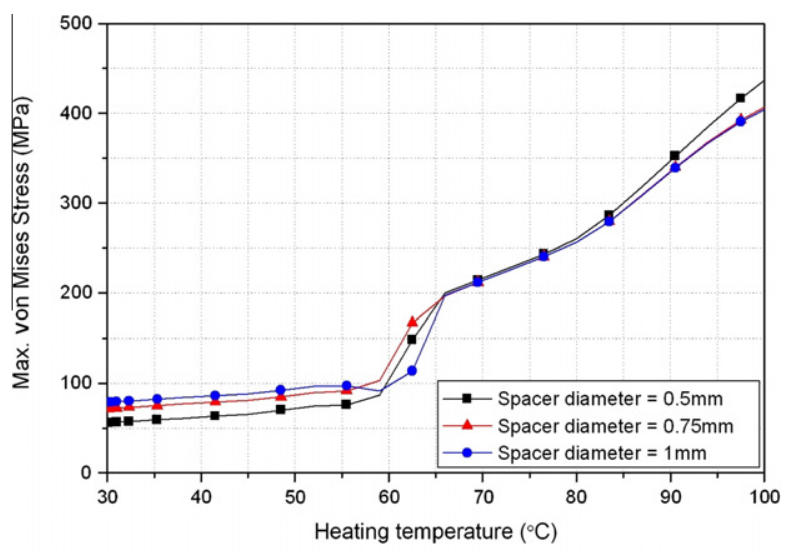

(a) Maximum von Mises stress

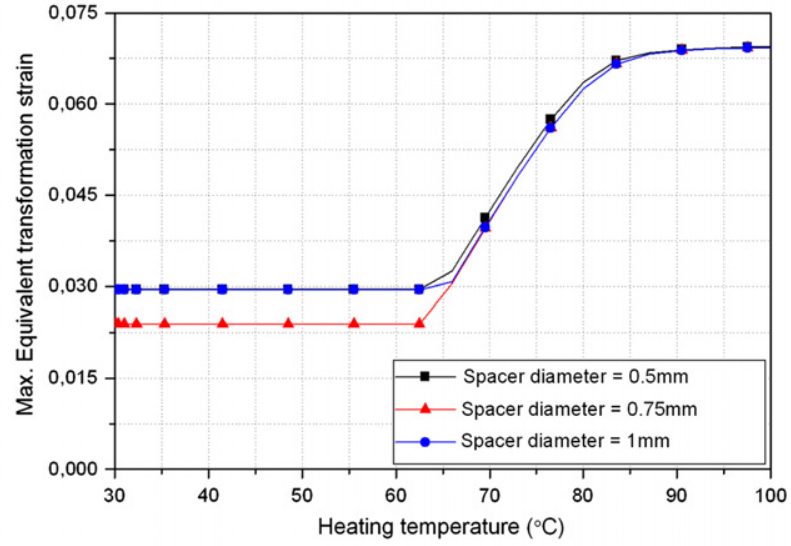

(b) Maximum equivalent transformation strain

Fig. 15. Maximum von Mises stress and equivalent transformation strain in the NiTi actuator as a function of heating temperature.

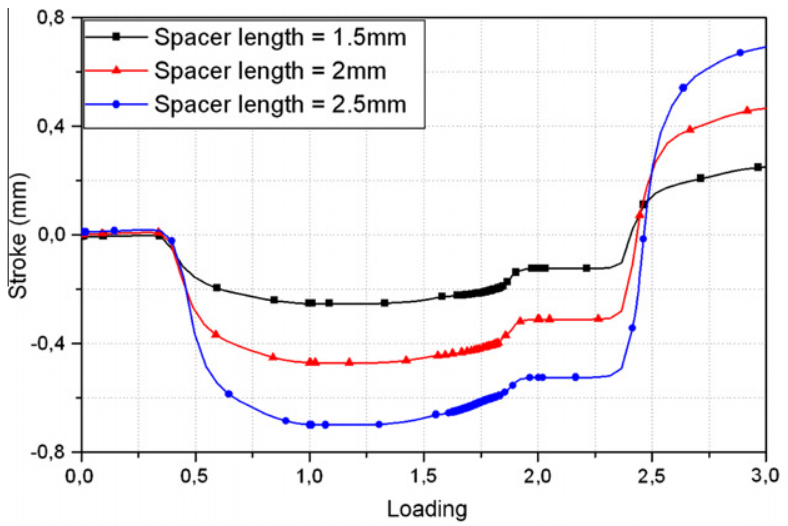

(a) Stroke

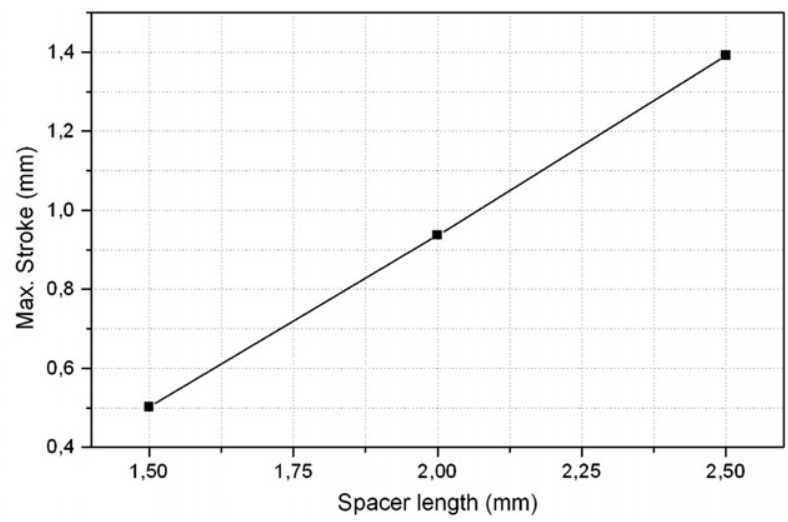

(b) Maximum stroke

Fig. 16. Stroke of the actuator during the steps $0-1,1-2$ and $2-3$. 


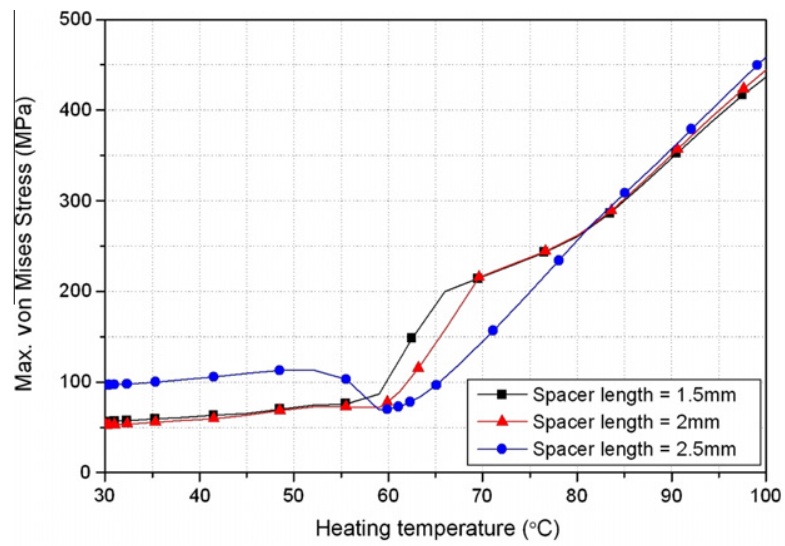

(a) Maximum von Mises stress

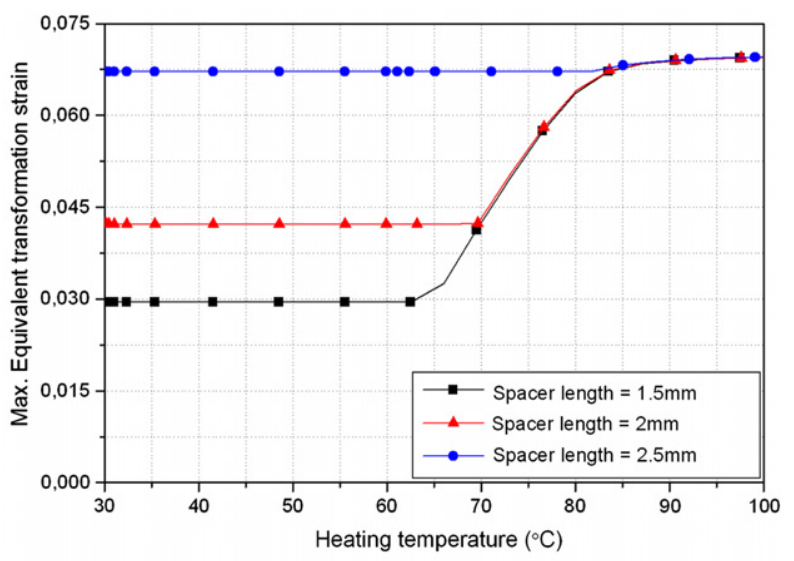

(b) Maximum equivalent transformation strain

Fig. 17. Maximum von Mises stress and equivalent transformation strain in the NiTi actuator as a function of heating temperature.

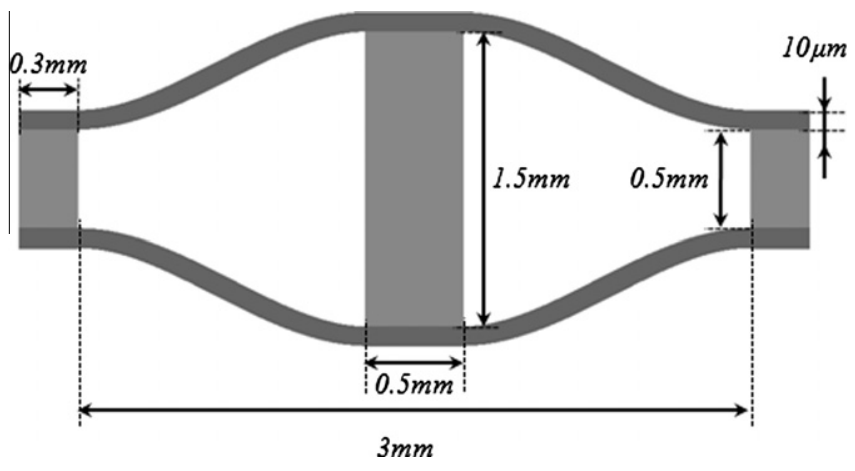

Fig. 18. Sketch of the optimal actuator, cross-section.

\subsection{Adopted parameters for the SMA actuator}

The parametric analysis allowed on the one hand, to study the thermomechanical behavior of the NiTi actuators, on the other hand to define an optimal design able to provide a large stroke for a minimal size actuator geometry. During this analysis, various parameters were selected in order to facilitate the manufacturing and assembly processes of the actuator. The adopted dimensions of the actuator geometry were presented in Fig. 18. To tend towards a really predictive model, a huge experimental work is necessary in order to improve the identification of all the physical parameters introduced in the model.

\section{Conclusion}

Finite Element simulation of an SMA actuator for a micropump was investigated in this paper. The sensitivity of actuator behavior to geometrical and material parameters was analyzed. This actuator is composed of two NiTi membranes. They are martensitic at room temperature. They are initially flats, and are bonded together with a crown in the exterior contour (exterior spacer), and with an intermediate spacer in silicon material. The principle is based on the heating of one or the other NiTi membrane, which allows the motion in both directions.

Finite Element analysis was adopted to simulate the actuator operating conditions and to investigate the design constraints for a displacement actuator. A phenomenological constitutive law describing the NiTi membranes behavior was adopted $[1,2]$. It is based on the assuming of expressions of Gibbs free-energy and dissipation function depending on a macroscopic control and internal variables. This model was implemented in the Abaqus FE code using the User MATerial subroutine (UMAT). The deflection and the stroke of the actuator were analyzed. For that, several simulations were carried out in the aim to exhibit the variation of the stroke with geometrical and material parameters, and to highlight the high influenced factors. 
Thus, it is concluded that results have shown reasonable agreement, providing validity of the constitutive model simulating the actuator behavior. The investigation of the various parameters influence in relation with the function of the actuator was an important step in this paper, the aim was to find acceptable values for these parameters and to improve effectively the performance. The parametric analysis allowed to define a set of material and geometrical parameters leading to an important stroke for a minimal global size geometry of the actuator. The stroke was obtained with a low heating of the NiTi membranes. During this analysis, some parameters were fixed in order to facilitate the manufacturing and assembly processes of the actuator. The obtained results prove the ability of the model to reproduce significant results when the actuator is submitted to thermal loading. Obtained results show the advantage to use the Finite Element analysis for Microelectromechanical systems (MEMS) with complex behavior.

The proposed model is of direct interest to design and to predict the lifespan of actuators submitted to thermal loading. Considering the literature about such actuators, the obtained results are encouraging. But, to tend towards a refined predictive model, additional experimental work is necessary in order to identify all the physical parameters. This work constitutes one more step to reinforce the method for studying the actuator behavior. However, the developed model can be broadened by adding of diverse physics, such as the interactions between the fluid/structure and microvalve effect in order to assess durability and long-term performance of actuator.

A second part of this study is in progress and dealing with the fabrication of this actuator. A discussion about NiTi material fabrication, parameters identification, and parameter sensitivity will be carried out. Experimental analysis of the actuator behavior under thermal loading and comparison between numerical simulations and experiments will be performed.

\section{Acknowledgements}

The authors acknowledge the financial support of the French National Agency for Research (ANR), through the MAFESMA French and European EUROCORES S3T Projects (Mathematical Algorithm Finite Element Shape Memory Alloys).

\section{References}

[1] Y. Chemisky, A. Duval, E. Patoor, T. Ben-Zineb, Constitutive model for shape memory alloys including phase transformation, martensitic reorientation and twins accommodation, Mechanics of Materials 43 (7) (2011) 361-376.

[2] A. Duval, M. Haboussi, T. Ben-Zineb, Modelling of localization and propagation of phase transformation in superelastic sma by a gradient nonlocal approach, International Journal of Solids and Structures 48 (13) (2011) 1879-1893.

[3] M. Frotscher, F. Kahleyss, T. Simon, D. Biermann, G. Eggeler, Achieving small structures in thin NiTi sheets for medical applications with water jet and micromachining: a comparison, Journal of Materials Engineering and Performance 20 (2011) 776-782.

[4] M. Kohl, E. Just, W. Pfleging, S. Miyazaki, Sma microgripper with integrated antagonism, Sensors and Actuators A: Physical 83 (1-3) (2000) $208-213$.

[5] A. Nisar, N. Afzulpurkar, B. Mahaisavariya, A. Tuantranont, Mems-based micropumps in drug delivery and biomedical applications, Sensors and Actuators B: Chemical 130 (2) (2008) 917-942.

[6] B. Jodway, M. Hülsmann, A comparative study of root canal preparation with NiTi-TEE and k3 rotary Ni-Ti instruments, International Endodontic Journal 39 (1) (2006) 71-80.

[7] G. Song, N. Ma, H.-N. Li, Applications of shape memory alloys in civil structures, Engineering Structures 28 (9) (2006) $1266-1274$

[8] W. Huang, Q. Liu, L. He, J. Yeo, Micro NiTi-Si cantilever with three stable positions, Sensors and Actuators A: Physical 114 (1) (2004) 118-122.

[9] A.D. Johnson, Vacuum-deposited tini shape memory film: characterization and applications in microdevices, Journal of Micromechanics and Microengineering 1 (1) (1991) 34.

[10] B. Hoxhold, S. Bnttgenbach, Batch fabrication of micro grippers with integrated actuators, Microsystem Technologies 14 (2008) 1917-1924.

[11] J.K. Paik, E. Hawkes, R.J. Wood, A novel low-profile shape memory alloy torsional actuator, Smart Materials and Structures 19 (12) (2010) 125014. 9pp.

[12] Y. Bellouard, Shape memory alloys for microsystems: a review from a material research perspective, Materials Science and Engineering: A 481-482 (0) (2008) 582-589.

[13] M. Tomozawa, H. Kim, S. Miyazaki, Shape memory behavior and internal structure of Ti-Ni-Cu shape memory alloy thin films and their application for microactuators, Acta Materialia 57 (2) (2009) 441-452.

[14] J. Busch, A. Johnson, Shape-memory alloys micro-actuator, US Patent, 5, 061, 914.

[15] R. Wolf, A. Heuer, Tini (shape memory) films silicon for mems applications, Journal of Microelectromechanical Systems 4 (1995) 206212.

[16] B.K. Kuila, M. Stamm, Block copolymer small molecule supramolecular assembly in thin film: a novel tool for surface patterning of different functional nanomaterials, Journal of Materials Chemistry 21 (2011) 14127-14134.

[17] M. Ehmann, S. Thienhaus, A. Ludwig, Micro-to nanostructured devices for the characterization of scaling effects in shape-memory thin films, Journal of Microelectromechanical Systems 21 (2010) 1264-1269.

[18] M.M. Ali, K. Takahata, Frequency-controlled wireless shape-memory-alloy microactuators integrated using an electroplating bonding process, Sensors and Actuators A: Physical 163 (1) (2010) 363-372.

[19] R.L. de Miranda, C. Zamponi, E. Quandt, Fabrication of tini thin film stents, Smart Materials and Structures 18 (10) (2009) 104010

[20] M. Kohl, K. Skrobanek, E. Quandt, P. Schloümacher, A. Schüssler, D. Allen, Development of microactuators based on the shape memory effect, Journal de Physique IV(C8) (3) (1995) 11871192

[21] M. Kohl, K. Skrobanek, S. Miyazaki, Development of stress-optimised shape memory microvalves, Sensors and Actuators A: Physical 72 (3) (1999) 243250.

[22] M. Bergamasco, P. Dario, F. Salsedo, Shape memory alloy microactuators, Sensors and Actuators A: Physical 21 (1-3) (1990) $253-257$.

[23] B. Bayer, S. Sanjabi, C. Baehtz, C. Wirth, S. Esconjauregui, R. Weatherup, Z. Barber, S. Hofmann, J. Robertson, Carbon nanotube forest growth on niti shape memory alloy thin films for thermal actuation, Thin Solid Films 519 (18) (2011) 6126-6129.

[24] Y. Fu, H. Du, W. Huang, S. Zhang, M. Hu, TiNi-based thin films in mems applications: a review, Sensors and Actuators A: Physical 112 (2-3) (2004) 395408.

[25] K.W. Oh, C.H. Ahn, A review of microvalves, Journal of Micromechanics and Microengineering 16 (5) (2006) 13-39.

[26] R. Krondorfer, Y.K. Kim, J. Kim, C.-G. Gustafson, T.C. Lommasson, Finite element simulation of package stress in transfer molded mems pressure sensors, Microelectronics Reliability 44 (12) (2004) 1995-2002.

[27] F. Krecinic, T.C. Duc, G.K. Lau, P.M. Sarro, Finite element modelling and experimental characterization of an electro-thermally actuated silicon-polymer micro gripper, Journal of Micromechanics and Microengineering 18 (6) (2008) 064007, http://dx.doi.org/10.1088/0960-1317/18/6/064007. 
[28] M. Zhu, P. Kirby, M. Wacklerle, M. Herz, M. Richter, Optimization design of multi-material micropump using finite element method, Sensors and Actuators A 149 (2009) 130-135.

[29] M.M. Teymoori, A.A. Sani, Design and simulation of a novel electrostatic micromachined pump for drug delivery applications, Sensors and Actuators A 117 (2005) 222-229.

[30] W.L. Benard, H. Kahn, A.H. Heuer, M.A. Huff, Thin film shape memory alloy actuated micropumps, Journal of microelectromechanical systems MEMS 7 (1998) 245-251.

[31] W.L. Benard, H. Kahn, A.H. Heuer, M.A. Huff, A titanium-nickel shape memory alloy actuated micropump, International Conference on Solid State Sensors and Actuators 1 (1997) 361-364.

[32] E. Makino, T. Mitsuya, T. Shibata, Fabrication of TiNi shape memory micropump, Sensors and Actuators A 88 (2001) 256-262.

[33] D. Xu, L. Wang, G. Ding, Y. Zhou, A. Yu, B. Cai, Characteristics and fabrication of NiTi/Si diaphragm micropump, Sensors and Actuators A: Physical 93 (1) (2001) 87-92.

[34] C.A. Ray, C.L. Sloan, A.D. Johnson, J.D. Busch, B.R. Petty, A silicon-based shape memory alloy microvalve, Materials Research Society Symposium Proceedings 276 (1992) 161166.

[35] B. Fan, G. Song, F. Hussain, Y.-c. shu, Shape-memory micropumps, Materials Transactions 43 (5) (2002) 10371044

[36] F. Sassa, Y. Al-Zain, T. Ginoza, S. Miyazaki, H. Suzuki, Miniaturized shape memory alloy pumps for stepping microfluidic transport, Sensors and Actuators B: Chemical 165 (1) (2012) 157-163.

[37] L. Gui, C.L. Ren, Exploration and evaluation of embedded shape memory alloy (sma) microvalves for high aspect ratio microchannels, Sensors and Actuators A: Physical 168 (1) (2011) 155-161.

[38] H. Iwanaga, H. Tobushi, H. Ito, Basic research on output power characteristics of a shape memory alloy heat engine, JSME International Journal Series I 31 (3) (1988) 634-637.

[39] J. Kan, K. Tang, G. Liu, G. Zhu, C. Shao, Development of serial-connection piezoelectric pumps, Sensors and Actuators A 144 (2008) $321-327$.

[40] C.R. de Lima, S.L. Vatanabe, A. Choi, P.H. Nakasone, R.F. Pires, E.C.N. Silva, A biomimetic piezoelectric pump: computational and experimental characterization, Sensors and Actuators A 152 (2009) 110-118.

[41] S.M. Ha, W. Cho, Y. Ahn, Disposable thermo-pneumatic micropump for biolab-on-a-chip application, Microelectronic Engineering 86 (2009) $1337-$ 1339.

[42] K. Junwu, Y. Zhigang, P. Taijiang, C. Guangming, W. Boda, Design and test of a high performance piezoelectric micropump for drug delivery, Sensors and Actuators A 121 (2005) 156-161.

[43] B. Fan, G. Song, F. Hussain, Simulation of a piezoelectrically actuated valveless micropump, Smart Materials and Structures 14 (2) (2005) 400.

[44] F. Auricchio, R.L. Taylor, Shape-memory alloys: modelling and numerical simulations of the finite-strain superelastic behavior, Computer Methods in Applied Mechanics and Engineering 143 (1-2) (1997) 175-194.

[45] F. Auricchio, R.L. Taylor, J. Lubliner, Shape-memory alloys: macromodelling and numerical simulations of the superelastic behavior, Computer Methods in Applied Mechanics and Engineering 146 (3-4) (1997) 281-312.

[46] B. Peultier, T. Ben-Zineb, E. Patoor, Macroscopic constitutive law of shape memory alloy thermomechanical behaviour. Application to structure computation by FEM, Mechanics of Materials 38 (2006) 510-524.

[47] W. Huang, On the selection of shape memory alloys for actuators, Materials \& Design 23 (1) (2002) 11-19.

[48] L. An, W. Huang, Y. Fu, N. Guo, A note on size effect in actuating niti shape memory alloys by electrical current, Materials \& Design 29 (7) (2008) 14321437.

[49] W. Huang, Modified shape memory alloy (sma) model for sma wire based actuator design, Journal of Intelligent Material Systems and Structures 10 (3) (1999) 221-231.

[50] N. Siredey, A. Hautcoeur, A. Eberhardt, Lifetime of superelastic Cu-Al-Be single crystal wires under bending fatigue, Materials Science and Engineering A 396 (2005) 296-301. 